As part of the 50th birthday celebration, we are arranging to reprint 12 papers, the editor's choice, which have appeared in previous issues of the fournal. They have been chosen partly to illustrate the range and scope of the fournal over the years and partly because they are or were important in their day. More significantly, they have been chosen because they exemplify some of the best in scientific writing and can all be read with great pleasure and all who wish to communicate their observations, their ideas, or their enthusiasms would do well to study them and learn from them.

We will publish one paper each month through the year and they will appear in the order in which they were originally published.

\title{
Skin cancer in the engineering industry from the use of mineral oil
}

by C N D Cruickshank and J R Squire

\section{(British Foumal of Industrial Medicine 1950;7:1-11)}

Cancer of the scrotum had been associated with chimney sweeps for nearly two centuries when Cruickshank and Squire published this paper, and more recent work by Southam and Wilson and by S A Henry had shown the connection with exposure to mineral oil in the cotton industry. Surprisingly little attention had been paid to the hazard of skin cancer in the engineering industry, however, despite the increased use of mineral oil, especially in bar automatic shops. Cruickshank and Squire drew their evidence from observations on men at work, from animal experiments, and from the Birmingham Cancer Registry. They were able to show a high prevalence of oil folliculitis and hyperkeratosis in machine tool workers and their experiments showed that the application of mineral oil to the skin of rabbits caused the production of benign tumours. The cancer registry yielded up 34 cases of scrotal cancer during the years $1938-48$ of whom 12 had been exposed to oil. Their findings suggested that toolsetters and toolmakers had a particularly high risk of developing the disease and they wrote that "the occurrence of ... scrotal cancer in this group ... was not an isolated event, and that others might be expected to arise." A quarter of a century later I was part of the group that investigated the incidence of scrotal cancer in the West Midlands, again using cancer registry data. We were able to confirm the prognostication in Cruickshank and Squire's paper; there was a sudden increase in the number of cases in 1956 that reached a peak in 1969. The numbers then declined with improvements in control measures, with the substitution of less carcinogenic oils, and a decrease in the number of workers exposed.

In their paper Cruickshank and Squire recommended the voluntary introduction of regular medical inspections of workers exposed to cutting oils. Their advice was ignored, however, with consequences that can only be guessed at. 